\title{
CONTROLE DA INFESTAÇÃO POR Ornithonyssus sylviarum (CANESTRINI E FANZAGO, 1877) (ACARI: MACRONYSSIDAE) EM POEDEIRAS COMERCIAIS UTILIZANDO EXTRATO DE Azadirachta indica
}

\author{
NILCE M. SOARES ${ }^{1}$; EDNA C. TUCCI ${ }^{2}$; ELIZABETH A. L. GUASTALLI ${ }^{1}$; HELENA YAJIMA ${ }^{1}$
}

\begin{abstract}
SOARES, N. M.; TUCCI, E. C.; GUASTALLI, E. A.L.; YAJIMA, H. [Control of Ornithonyssus sylviarum (Canestrini and Fanzago, 1877) (Acari: Macronyssidae) infestation in commercial laying hens by using Azadirachta indica extract]. Controle da infestação por Ornithonyssus sylviarum (Canestrini e Fanzago, 1877) (Acari: Macronyssidae) em poedeiras comerciais utilizando extrato de Azadirachta indica. Revista Brasileira de Parasitologia Veterinária, v. 17, n. 4, p. 175-178, 2008. Unidade Laboratorial de Patologia Avícola, Centro Avançado de Pesquisa Tecnológica do Agronegócio Avícola, Instituto Biológico, Avenida Gaspar Ricardo nº 1700, Bastos, SP 17690-000, Brasil. E-mail: updbastos@biologico.sp.gov.br

This study evaluated the effectiveness of a neem extract-based product to control $O$. sylviarum infestations in commercial laying hens. The birds were divided in 3 groups, which received 2, 3, or 4 applications of the product at 7 day intervals. The results obtained allow the conclusion that the neem extract at $2 \%$ is effective to control infestations by $O$. sylviarum, and at least 3 sprays of the product are required weekly for an effective control of the parasite.
\end{abstract} \section{RESUMO}

KEY WORDS: Haematophagous mite, laying hens, ectoparasites, northern fowl mite.

O presente trabalho teve por objetivo avaliar a eficácia de um produto à base de extrato de nim, no controle da infestação por $O$. sylviarum em poedeiras comerciais. O experimento foi realizado observando-se a eficácia do extrato de nim a 2\% após duas, três e quatro aplicações com intervalo de sete dias. Os resultados obtidos permitem concluir que o extrato de nim a $2 \%$ é efetivo no controle da infestação por $O$. sylviarum, sendo necessárias pelo menos três pulverizações semanais do produto para que haja o controle efetivo do parasito.

PALAVRAS-CHAVE: Ácaros hematófagos, galinhas poedeiras, ectoparasitas.

\section{INTRODUÇÃO}

Os modernos sistemas de produção de ovos para consumo implicam na criação das aves em confinamento, com alta densidade de animais nas instalações, o que contribui para proliferação de importantes ectoparasitas de aves, principalmente os ácaros hematófagos.

${ }^{1}$ Unidade Laboratorial de Patologia Avícola, Centro Avançado de Pesquisa Tecnológica do Agronegócio Avícola, Instituto Biológico, Avenida Gaspar Ricardo $\mathrm{n}^{\circ}$ 1700, Bastos, SP 17690-000, Brasil. E-mail: updbastos@biologico.sp.gov.br

${ }^{2}$ Centro de Sanidade Animal, Instituto Biológico, São Paulo, SP. VANEY, 1979).
Os ácaros hematófagos são parasitas obrigatórios das galinhas de criação industrial e de outras espécies de aves domésticas e silvestres. Nos Estados Unidos as perdas da indústria avícola determinadas pelo parasitismo destes ácaros foram superiores a 76 milhões de dólares na década de 70 (DE

No Brasil foram assinaladas três espécies de ácaros hematófagos parasitando as galinhas de postura: Dermanyssus gallinae, Ornithonyssus sylviarum e O. bursa. Entretanto, somente as duas primeiras espécies ocorrem em aviários industriais de postura (FONSECA, 1938; REIS, 1939; FACCINI; MASSARD, 1974; PEREIRA et al., 1977; FACCINI, 1987; TUCCI, et al., 1997).

Ornithonyssus sylviarum, também denominado ácaro do norte ou ácaro da pena, característico de todas as regiões temperadas do mundo representando uma séria praga para a avicultura de postura (LANCASTER; MEISH, 1986). No estado de São Paulo está bem distribuído, sendo encontrado em 48,8\% das granjas visitadas por Tucci et al. (1997).

As populações de $O$. sylviarum crescem rapidamente e uma geração pode ser completada em cinco a sete dias, podendo viver além de seis semanas na ausência de seus hospedeiros. Permanece no hospedeiro durante todo seu ciclo de vida, formando colônias no ventre e ao redor da cloaca da ave. As penas desta região ficam escurecidas conferindo às aves um aspecto de sujo. Quando um lote de aves apresenta 
um grau de infestação muito elevado, o ácaro é encontrado também nas fezes que ficam em baixo das gaiolas, nos ovos produzidos e infestando o trabalhador que cuida das aves, causando irritação, alergias e dermatites. As aves infestadas apresentam-se estressadas, com perda de peso, queda de produção de ovos, debilidade podendo ser acometidas por doenças secundarias, anemia em vários graus, chegando às vezes até a morte (MATTHYSSE et al.,1974; DE VANEY, 1978; LANCASTER JR; MEISH.,1986; GUIMARÃES et al., 2001).

Desde o início do século passado, vários produtos como DDT, organofosforados, organoclorados, carbamataos e piretroides vêm sendo utilizados na tentativa de controle destes ácaros, entretanto o uso destas substâncias pode determinar alto nível de toxicidade para as aves. Além disto, oferece grande desvantagem, como risco à saúde do aplicador e ao consumidor do produto final por apresentarem resíduos e poluindo o meio ambiente (GUIMARÃES, 2000).

A utilização de produtos naturais nas explorações comerciais para produção de alimentos destinados ao consumo humano vem conquistando mercado e a preferência dos produtores e consumidores, não apenas pela redução do uso de agrotóxicos, como pela adoção de praticas que não causem danos ao meio ambiente (MARTINEZ, 2002).

O NIM, Azadirachta indica, planta originada da Índia, trazida para o Brasil em 1992, tem papel fundamental neste processo, através do seu histórico de utilização popular, desencadeando uma serie de trabalhos que fazem desta espécie a planta inseticida mais estudada e utilizada atualmente (BOGORNI; VENDRAMIM, 2001).

Azadirachta indica possui em sua composição diversas substâncias com ação inseticida e acaricida, que controlam muitas espécies de artrópodes atuando, de um modo geral, por sua ação antialimentícia, repelente, por atuar na ecdise, diminuir a postura e pela ação ovicida. Não existe registro de toxicidade destas substâncias para humanos (MULLA; SU, 1999; MARTINEZ, 2000).

Face ao exposto e visando fornecer subsídios para o controle deste ectoparasita, foi elaborado o presente estudo com o objetivo de avaliar a ação do extrato de NIM (produto comercial denominado Nimkol-LS ${ }^{\circledR}$ ), no controle do ácaro hematófago $O$. sylviarum em galinhas poedeiras, analisando-se a eficácia e o número de aplicações necessárias para o controle.

\section{Local}

\section{MATERIAL E MÉTODOS}

O experimento foi conduzido no município de Bastos (la-

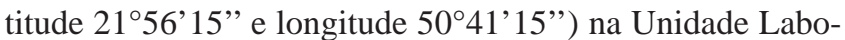
ratorial de Patologia Avícola do Instituto Biológico. As aves foram alojadas em galpão convencional de postura comercial, com cobertura de telhas de barro e com duas fileiras de gaiolas de arame galvanizado, sobrepostas de cada lado, na proporção de duas aves por gaiola. Durante o período experimental as aves receberam ração e água à vontade, sendo que a ração foi distribuída duas vezes ao dia para evitar o desperdício. O fotoperíodo foi de 16:8 (C:E).
Aves

Foram utilizadas galinhas de postura comercial da linhagem Hy line e Lohmann com 25 semanas de idade no início do experimento, naturalmente infestadas por $O$. sylviarum.

\section{Infestação}

Para avaliação dos níveis de infestação por O. sylviarum, as aves foram examinadas individualmente, antes e após os tratamentos, para contagem ou estimativa do nível de segundo critério estabelecido por DE VANEY (1979): 0- sem ácaros,1- muito baixa (1 a 10 ácaros/ave); 2- fraca (11 a 100); 3- moderada (101 a 1000); 4- forte(1001 a 10.000) e 5- extra forte(mais de 10.000).

\section{Produto}

Foi utilizado o produto comercial Nimkol-LS $\AA$, composto de extrato de Folhas de NIM a 20\%, em veículo líquido inerte, e óleo de Semente de NIM (NeemAzal T/S a 10\% contendo 1000 ppm de Azadirachtina).

O produto a base de NIM foi diluído a $2 \%$ e adicionado $0,1 \%$ de óleo mineral e $0,1 \%$ de detergente. A aplicação foi feita por pulverização, utilizando-se bomba costal, até que as penas das aves ficassem totalmente molhadas.

Foram utilizados quatro grupos experimentais com 12 aves cada: Grupo I: aves tratadas com duas aplicações do produto no $1^{\circ} \mathrm{e} 7^{\circ}$ dias; Grupo II: aves tratadas com três aplicações do produto no $1^{\circ}, 7^{\circ}$ e $14^{\circ}$ dias; Grupo III: aves tratadas com quatro aplicações do produto no $1^{\circ}, 7^{\circ}, 14^{\circ}$ e $21^{\circ}$ dias. Grupo IV: aves tratadas somente com água (grupo controle). A infestação das aves por $O$. sylviarum foi avaliada antes da aplicação do produto e semanalmente por 84 dias.

O delineamento estatístico foi inteiramente casualizado. Os resultados foram transformados em raiz de $\mathrm{x}+0,5$, submetidos à análise de Variância (ANOVA) e ao teste de Tukey para comparação de médias, ao nível de 5\% de significância.

\section{RESULTADOS}

Todos os grupos tratados diferiram estatisticamente do grupo controle, o qual apresentou nível de infestação igual ou superior a dois em todas as observações realizadas (Tabela 1). Verificou-se que os grupos de aves que receberam três e quatro aplicações, não diferiram estatisticamente entre si, apresentando níveis de infestação igual a zero, 14 dias após o início do tratamento, permanecendo assim até o final das observações. O grupo que recebeu duas aplicações sofreu uma diminuição na população dos ácaros, estatisticamente significante em relação ao grupo controle, na maioria das semanas observadas, entretanto apesar da redução, os ácaros permaneceram presentes até o final das observações.

\section{DISCUSSÃO}

O presente trabalho demonstrou que o produto a base de NIM foi eficaz no controle de $O$. sylviarum, agindo por um longo período, com redução da população superior a 50\% até 
Tabela 1. Nível de infestação por O. sylviarum em aves tratadas com extrato de NIM a 2\%, nos diferentes dias após as aplicações.

\begin{tabular}{|c|c|c|c|c|c|c|c|c|c|c|c|c|c|}
\hline \multirow{3}{*}{$\begin{array}{l}\text { Número de } \\
\text { Aplicações }\end{array}$} & \multicolumn{13}{|c|}{ Nível de infestação* } \\
\hline & \multicolumn{13}{|c|}{ Dias após o tratamento } \\
\hline & -1 & +7 & +14 & +21 & +28 & +35 & +42 & +49 & +56 & +63 & +70 & +77 & +84 \\
\hline 2 & $2,9 \mathrm{a}$ & $2,1 \mathrm{a}$ & $0,9 \mathrm{~b}$ & $1,2 \mathrm{~b}$ & $1,8 \mathrm{~b}$ & $2,05 \mathrm{a}$ & $1,9 \mathrm{a}$ & $1,09 \mathrm{~b}$ & $1 \mathrm{~b}$ & $1 \mathrm{~b}$ & $1 \mathrm{~b}$ & $1 \mathrm{~b}$ & $1 \mathrm{~b}$ \\
\hline 3 & $2,5 \mathrm{a}$ & $2,1 \mathrm{a}$ & $1,6 \mathrm{a}$ & $0 \mathrm{c}$ & $0 \mathrm{c}$ & $0 \mathrm{~b}$ & $0 \mathrm{~b}$ & $0 \mathrm{c}$ & $0 \mathrm{c}$ & $0 \mathrm{c}$ & $0 \mathrm{c}$ & $0 \mathrm{c}$ & $0 \mathrm{c}$ \\
\hline 4 & $2,5 \mathrm{a}$ & $1,6 \mathrm{a}$ & $1,6 \mathrm{a}$ & $0 \mathrm{c}$ & $0 \mathrm{c}$ & $0 \mathrm{~b}$ & $0 \mathrm{~b}$ & $0 \mathrm{c}$ & $0 \mathrm{c}$ & $0 \mathrm{c}$ & $0 \mathrm{c}$ & $0 \mathrm{c}$ & $0 \mathrm{c}$ \\
\hline Controle & $2,6 a$ & $2,2 \mathrm{a}$ & $2,2 \mathrm{a}$ & $2,3 \mathrm{a}$ & $2,4 \mathrm{a}$ & $2,6 \mathrm{a}$ & $2,2 \mathrm{a}$ & $2,0 \mathrm{a}$ & $2,1 \mathrm{a}$ & $2,2 \mathrm{a}$ & $2,2 \mathrm{a}$ & $2,1 \mathrm{a}$ & $2,1 \mathrm{a}$ \\
\hline CV (\%) & 13,3 & 14,3 & 14,0 & 15,0 & 11,5 & 11,7 & 11,9 & 8,9 & 8,4 & 9,3 & 9,3 & 13,4 & 11,8 \\
\hline
\end{tabular}

*medias seguidas da mesma letra não diferem entre si ao nível de 5\% de significância (Teste de Tukey)

CV - Coeficiente de Variação

o final do experimento. A não eliminação completa dos ácaros no grupo que recebeu duas aplicações, pode estar associada ao fato do produto não agir sobre os ovos ou por não possuir uma ação residual, não agindo, desta forma, sobre as larvas que emergiram dos ovos remanescentes. Assim, com um maior número de aplicações obteve-se uma melhor eficácia no controle, com 100\% de mortalidade dos ácaros em 21 dias, nos experimentos com três e quatro aplicações do produto.

Trabalhos semelhantes foram realizados por Larramendy et al.(2003), em Cuba, com produtos a base de NIM no controle dos ectoparasitas Megninia gynglimura e Menopon gallinae. Os autores realizaram três tratamentos, com intervalos de 15 e 30 dias obtendo $100 \%$ de controle dos parasitos, 45 dias após o tratamento inicial. Na Índia, Das et al. (1993) obtiveram sucesso no controle de piolhos com extratos de plantas. Os autores verificaram o efeito inseticida de extratos de Cedrus deodara, A. indica e Embelia ribes, em aves tratadas com os extratos por pulverização. Constataram que as concentrações de 1:10 a 1:50 determinaram $100 \%$ de mortalidade de M. gallinae e Lipeurus caponis, em 24 horas após o tratamento.

$\mathrm{Na}$ avicultura de postura, os riscos de contaminação dos ovos por substâncias químicas é muito alto (TUCCI et al. 1997). O uso do NIM, assim como de outras substâncias naturais, possui a vantagem de não deixar resíduos nos produtos de origem animal, não poluírem nem determinarem riscos ao meio ambiente, à saúde do aplicador e do consumidor final dos produtos. Desta forma o uso do NIM representa mais uma alternativa no controle do $O$. sylviarum e vem ao encontro das necessidades de uma avicultura moderna, na busca por alimentos seguros mais saudáveis, isentos de defensivos químicos.

O Brasil é um dos principais países do mundo na exportação de produtos avícolas, devido à alta eficiência na produção e excelente qualidade de seu produto final. Desta forma o uso do NIM no controle de O. sylviarum, vem ao encontro das necessidades de uma avicultura moderna, na busca por alimentos seguros, mais saudáveis e isentos de defensivos agrícolas , que atendam as necessidades do mercado internacional.

\section{REFERÊNCIAS BIBLIOGRÁFICAS}

ABBOTT, W.S. A method of computing the effectiveness of an insecticide. Journal of Economic Entomology, v. 18, p. 265-267, 1925.
BOGORNI, P.C.; VENDRAMIM, J.D. Nim e outras plantas inseticidas. In: VENDRAMIM, J.D.; BOGORNI, P.C.; ABREU JÚNIOR, H. (Org). Nim, o protetor natural múltiplo. Piracicaba: ESALQ, 2001. p.1-5.

DAS, S.S.; BHATIA, B.B.; KUMAR, A. Efficacy of Pestoban-D against common poultry lice. Indian Journal of Veterinary Research. v. 2, n. 2, p. 25-26, 1993.

DeVANEY, J.A. A survey of poultry ectoparasite problems and their research in the United States. Poultry Science, v. 57, n. 5, p. 1217-1220, 1978.

DeVANEY, J.A. The effects of the nothem fowl mite Ornithonyssus sylviarum on egg production and body weith of cage white leghorn hens. Poultry Science, v. 58, n. 1, p. 191-194, 1979.

FACCINI J.L.H. Ácaros hematófagos: parasitos de aves de postura (Gallus gallus) no Brasil. Diversificação, biologia e controle. Arquivo Fluminense de Medicina Veterinária, v. 2, n. 1, p. 29-31, 1987.

FACCINI J.L.H.; MASSARD, C.L. Nota sobre a ocorrência de Ornithonyssus sulviarum (Canestrini \& Fanzago) (Mesostigmata: Mascronyssidae) em Gallus gallus no Brasil. Arquivos da Universidade Federal Rural do Rio de Janeiro, v. 4, n. 1, p. 39-40. 1974.

FONSECA, F. Notas de Acarologia. Boletim Biológico, v. 3, n. 3, p. 132, 1938.

GUIMARÃES J.H. Ectoparasitas e outros artrópodes importantes para a indústria avícola brasileira. In: BERCHIERI JÚNIOR, A.; MACARI, M. (Eds.) Doenças das Aves. Campinas: FACTA, 2000. p. 413-22.

GUIMARÃES, J.H.; TUCCI, E.C.; BARROS-BATTESTI D.M. Ectoparasitas de importância veterinária. $1^{\text {a }}$ ed. São Paulo: Plêiade/FAPESP, 2001. 218p.

LANCASTER JR., J.L.; MEISH, M.V. Arthropods in livestock and poultry production. 1st ed., Chischester: Elly Horwood Limited, 1986, 402p.

MARTINEZ, S.S. O Nim - Azadirachta indica - natureza, usos múltiplos, produção. Londrina: Instituto Agronômico do Paraná, 2002. 142p.

MATTHYSSE, J.G.; JONES, C.J.; PURNASIRI, A. Development of northern fowl mite Ornithonyssus sylviarum (Canestrini \& Fanzago) (Acarina: Dermanyssidae). Populations on chickens, effects on the host, and 
immunology. Search Agriculture (Entomology 13), Cornell University, v. 4, p. 1-39. 1974.

MULLA, M.S.; SU, T. Activity and biological effects of neem products against arthropods of medical and veterinary importance. Journal of American Mosquitoe Control Association, v. 15, n. 2, p. 133-152, 1999.

NEVES, B.P. Nim, princípios e aplicações como defensivo agrícola. In: CONGRESSO BRASILEIRO DE DEFENSIVOS AGRÍCOLAS NATURAIS. FORTALEZA,1,2000, Fortaleza. Anais...Fortaleza: CBDA, 2000., p.5-6.

PEREIRA, M.C.; OBA, M.S.P.; SCHUMAKER, T.T.S. Ornithonysus sylviarum (Canestrini e Fanzago 1877) (Mesostignata: Macronyssidae) em Gallus gallus domesticus (L.) no Estado de São Paulo, Brasil. Revista da Faculdade de Medicina Veterinária e Zootecnia da Universidade de São Paulo, v. 14, n. 1, p. 243251, 1977.

REIS, J. Alguns parasitas de Gallus gallus (L.) verificados em São Paulo. Arquivos do Instituto Biológico, São Paulo, v. 10, p. 147-153, 1939.

TUCCI, E.C.; GUIMARÃES, J.H.; BRUNO, T.V.; GAMA, N.M.S.Q.; SANTOS, A.M.M. Ocorrência de ácaros hematófagos em aviários de postura no Estado de São Paulo, Brasil. Revista Brasileira de Parasitologia Veterinária, v. 5, n. 2, p. 95-102, 1997.

LARRAMENDY, R.; SZCZYPEL, B.; PEREZ, A.; GONZALES, A.; ESTRADA, J. Efectividad de distintos derivados del arbol del Nim (Azadirachta indica A. Juss) en gallinas naturalmente infestadas con ectoparasitos. Revista Cubana de Ciencia Avícola, v. 28, n. 1, p. 1-6, 2003.

Recebido em 26 de maio de 2006

Aceito para publicação em 30 de dezembro de 2008. 